\title{
Атомная конфигурация и зарядовое состояние водорода на дислокациях в кремнии
}

\author{
(C) Н.В. Высотский, А.С. Лошаченко, О.Ф. Вывенко
}

Санкт-Петербургский государственный университет, 198504 Санкт-Петербург, Россия

E-mail: mrdestroyed@yandex.ru; a.loshachenko@spbu.ru; vyvenko@gmail.com

(Получена 26 июля 2016 г. Принята к печати 10 августа 2016 г.)

Исследовалось влияние введения водорода на колебательные спектры и электрофизические свойства образцов с дислокационными сетками (ДС) на интерфейсе сращенных пластин кремния. Для увеличения чувствительности измерений и выделения сигнала ДС в спектрах комбинационного рассеяния использовались образцы в виде стандартных тонких фольг, применяемых в просвечивающей электронной микроскопии. В образцах с дислокационными сетками был зарегистрирован пик комбинационного рассеяния $2000 \mathrm{~cm}^{-1}$, который сохранялся после отжига при $T=500^{\circ} \mathrm{C}$ и не наблюдался в контрольных образцах. Сопоставление экспериментальных данных с имеющимися теоретическими расчетами позволило приписать обнаруженный пик к $H^{0}$ в центре $\mathrm{Si}-\mathrm{Si}$ связи, который является метастабильным в идеальной решетке, но стабилизируется в окрестности дислокации.

DOI: 10.21883/FTP.2017.03.44198.8378

\section{1. Введение}

При внедрении водорода в кристаллическую решетку кремния он активно взаимодействует с различными нарушениями ее структуры: примесями, собственными точечными и протяженными дефектами. Результатом такого взаимодействия во многих случаях является уменьшение или даже полное исчезновение (пассивация) электрической активности имеющихся дефектов [1-4]. С другой стороны, присутствие водорода может быть обнаружено методами ИК оптического поглощения (ИКОП) и комбинационного рассеяния (КР) за счет появления специфических локальных колебательных мод в соответствующих спектрах. Сопоставление теоретических расчетов с экспериментальными данными позволяет с большой точностью идентифицировать найденные частоты колебательных мод и отождествить их с различными атомными конфигурациями в решетке, в том числе и с водородными комплексами.

К настоящему времени большинство атомных конфигураций водорода, как для регулярной решетки, так и для его комплексов с точечными дефектами, считаются хорошо определенными. Так, в случае решетки, близкой к идеальной, установлена возможность существования двух водородных конфигураций, соответствующих локальным минимумам потенциальной энергии. Наименьшей энергией обладает атом водорода в составе молекулы $H_{2}$, находящейся в межузельном положении в центре тетраэдрической пустоты [5], соответствующей колебательной моде $3600 \mathrm{~cm}^{-1}$ [6,7]. Вторая конфигурация характеризуется линией КР в окрестности $2000 \mathrm{~cm}^{-1}$ и соответствует атому $H$ в центре ковалентной связи между двумя атомами кремния (bond-centered, $\mathrm{BC}$-положение). Данная конфигурация является метастабильной, так как, согласно теории, имеет энергию более чем на 1 эВ выше, чем упомянутая молекулярная конфигурация. Эксперименты по низкотемпературной имплантации протонами [8-10] подтверждают существование ВС-конфигурации в регулярной решетке, а также демонстрируют ее неустойчивость при температурах выше $200 \mathrm{~K}$, при которых $H$ переходит в другие более устойчивые конфигурации.

Экспериментальные данные по исследованию экзодиффузии водорода из пластически деформированного кремния [11,12] свидетельствуют о повышении энергии связи водорода с кристаллической решеткой у дислокаций, но не дают информации о его атомарной конфигурации в этом случае. Из теоретических расчетов $[13,14]$ ожидается значительное увеличение энергии связи, а следовательно, и повышение стабильности для атомарной ВС-конфигурации водорода в окрестности дислокаций при неизменности энергии связи молекулярной формы водорода. Это делает атомарную форму водорода энергетически более выгодной, чем молекулярную, уже в областях упругих напряжений на некоторых расстояниях от ядра дислокации. Расчеты $[13,15]$ для различных дислокационных ядер предсказывают возможность существования по крайней мере нескольких стабильных атомарных конфигураций водорода, чья энергия связи выше, чем у молекулярной формы. Однако до настоящей работы прямых экспериментальных подтверждений этим предсказаниям, насколько нам известно, не было.

Такая ситуация, на наш взгляд, объясняется тем, что в большинстве предшествующих работ по исследованию свойств дислокационного кремния использовались пластически деформированные образцы, в которых максимально достижимая плотность дислокаций относительно мала (как правило, не более $10^{9} \mathrm{~cm}^{-2}$ ). В связи с чем доля водорода, связанного с дислокациями, по нашему предположению, также является недостаточной для его регистрации с помощью КР или ИКОП. 
Значительно бо́льшая локальная плотность дислокаций может быть получена путем сращивания разориентированных относительно друг друга пластин (СП) кремния. При таком процессе на интерфейсе СП образуется совершенная дислокационная сетка (ДС) с плотностью дислокаций на 1-2 порядка выше, чем при пластической деформации. Недавние исследования показали, что при гидрогенизации данных образцов с помощью жидкохимического травления (ЖХТ) в растворе $H F$ при комнатной температуре водород активно сегрегирует на ДС, которая препятствует дальнейшему проникновению водорода в объем образца [16]. При этом водород может быть экстрагирован с ДС с помощью приложения электрического поля при температурах выше $70^{\circ} \mathrm{C}[17]$.

В настоящей работе с помощью специально приготовленных образцов нам впервые удалось при комнатной температуре зарегистрировать линию КР, соответствующую моноатомному водороду, продемонстрировать, что источник этого сигнала неразрывно связан с водородом на ДС, а также установить зарядовое состояние водорода, захваченного на ДС.

\section{2. Образцы и техника эксперимента}

Исходные пластины для исследований представляли собой кремниевые СП с ориентацией поверхности (001). Угол относительного разворота двух пластин при сращивании составлял около $3^{\circ}$, угол наклона $\sim 0.5^{\circ}$. Глубина залегания интерфейса СП - 150-180нм от поверхности. По данным просвечивающей электронной микроскопии (ПЭМ) [18,19] интерфейс сращенных пластин состоял из сетки винтовых дислокаций с периодом $7-8$ нм и рядов $60^{\circ}$ дислокаций, отстоящих друг от друга на расстоянии около 25-30нм. Сращенные пластины были изготовлены компанией SoiTech с применением технологии SmartCut ${ }^{\circledR}$, имели один тип проводимости и были легированы в случае $p$-типа бором $\left(10^{15} \mathrm{~cm}^{-3}\right)$, в случае $n$-типа - фосфором $\left(3 \cdot 10^{14} \mathrm{~cm}^{-3}\right)$.

Для оценки количества водорода в окрестности ДС и его зарядового состояния были проведены измерения вольт-фарадных характеристик (ВФХ) шоттки-диодов, изготовленных на основе пластин $p$-типа. Титановый контакт для создания барьера Шоттки наносился методом электронно-лучевого распыления в вакуумной камере. Формирование омического контакта с тыльной стороны образца производилось путем втирания InGa эвтектики. В качестве контрольного образца использовался аналогичный диод $p$-типа, не содержащий интерфейса (тыльная сторона). Гидрогенизация происходила перед напылением контакта в процессе выдержки образцов в растворе $\mathrm{HF}: 10 \mathrm{H}_{2} \mathrm{O}$ не менее 1 мин. Дислокационная структура исследовалась в просвечивающем электронном микроскопе Zeiss Libra $200 \mathrm{FE}$.

Для повышения чувствительности измерений спектров КР из исходных пластин были приготовлены фоль- ги по стандартной процедуре, используемой в ПЭМ. На первом этапе вырезанные из исходных пластин шайбы диаметром 3 мм механически шлифовались с тыльной стороны до толщины около $0.15 \mathrm{MM}$, затем в растворе полирующего травителя $\mathrm{HF}: 7 \mathrm{HNO}_{3}$ изготавливалась фольга толщиной 1-10 мкм, и, наконец, финальное утонение осуществлялось с помощью ионного (Ar) травления до появления отверстия в центре. В процессе указанного травления в кислотном растворе одновременно происходила и гидрогенизация. В качестве контрольного образца использовались фольги без интерфейса.

КР спектры были получены на спектрометре Senterra при возбуждении $\mathrm{Nd}$-лазером с длиной волны 532 нм и диаметром луча $\sim 6$ мкм. Диапазон измерений: $400-4000 \mathrm{~cm}^{-1}$.

\section{3. Результаты}

Схематическое изображение образца, используемого для КР измерений, приведено на рис. 1, $a$. Как показано на рисунке, вблизи края центрального отверстия фольга имеет клиновидную форму, при этом непосредственно
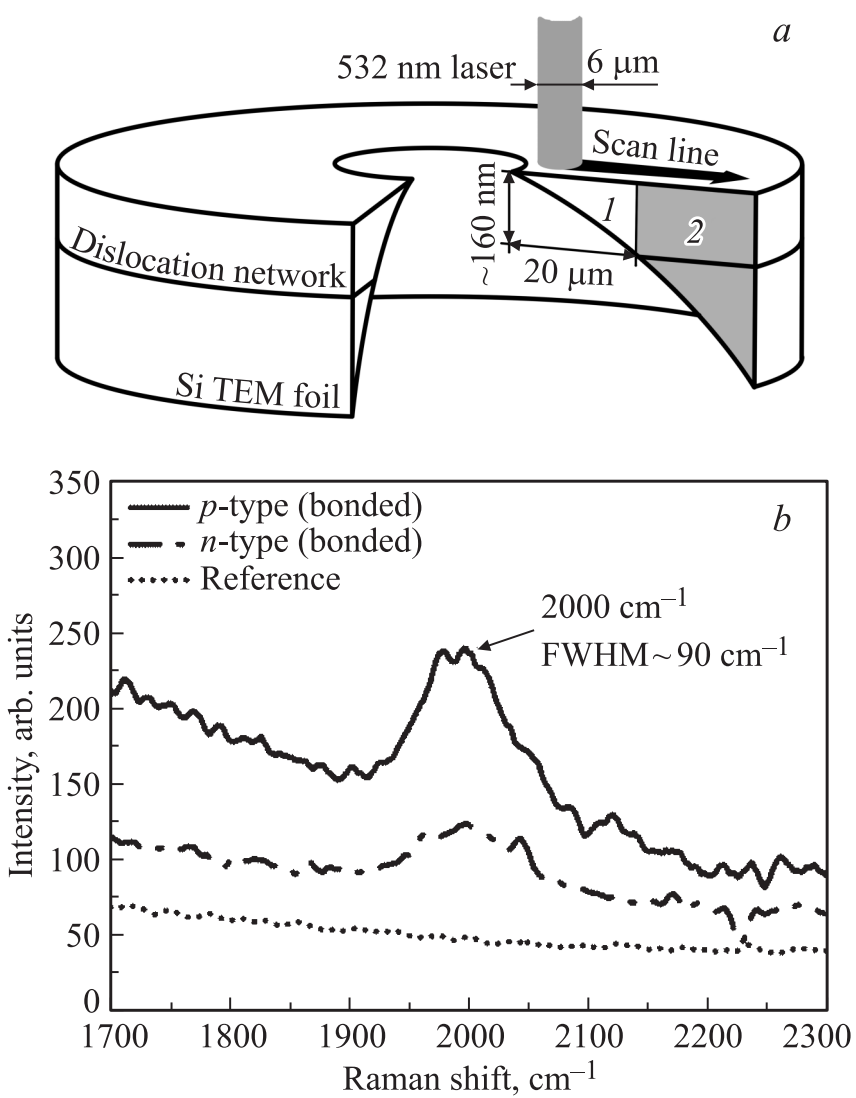

Рис. 1. $a-$ схематическое изображение ПЭМ фольги с ДС для исследований КР. Вертикальная линия между областями 1 и 2 показывает проекцию на исследуемую поверхность линии выхода ДС на нижнюю поверхность фольги. $b-$ сигнал КР в окрестности $2000 \mathrm{~cm}^{-1}$ для контрольного образца и для дислокационных образцов $p$ - и $n$-типов. 
у края имеется область, в которой ДС отсутствует. Эта область обозначена на рис. 1, $a$ цифрой 1. Спектры КР записывались от точки к точке в радиальном направлении по лицевой стороне фольги. Сканирование начиналось с первой области и заканчивалось в области с ДС, которая обозначена на рисунке цифрой 2.

Для бездислокационной фольги по всей области сканирования никакой особенности в спектре обнаружено не было, в то время как в фольгах с ДС обоих типов проводимости, начиная с границы областей 1 и 2 , наблюдался пик КР $2000 \mathrm{~cm}^{-1}$, который, согласно теоретическим расчетам, отвечает атомарному водороду в ВС. Обнаруженный пик характеризовался аномально большой полушириной, которая составляла около $90 \mathrm{~cm}^{-1}$. На рис. $1, b$ представлены наиболее интенсивные сигналы пика $2000 \mathrm{~cm}^{-1}$ для дислокационных образцов и один из спектров, полученных на контрольных фольгах.

На рис. 2 представлена комбинация экспериментальных данных, полученных методом КР и ПЭМ. Фоном данного рисунка служит фотография фольги в оптическом микроскопе (вид сверху), где отчетливо видно темное пятно центрального отверстия (слева), а также полосы, возникающие вследствие интерференции падающего света. Линия сканирования лазерным лучом отмечена на рис. 2 черным цветом с указанием масштабных меток. Белая гребенка на рис. 2 схематически изображает профиль выхода дислокационной сетки на поверхность фольги, что соответствует границе областей 1 и 2 на рис. 1, $a$. Данный профиль был определен по серии снимков в ПЭМ, один из которых приведен на вставке рис. 2.

Зависимость интенсивности пика $2000 \mathrm{~cm}^{-1}$ от места сканирования представлена сплошной кривой на рис. 2 . Как видно из рисунка, в наиболее тонком месте пленки, у самого отверстия, где нет ДС, сигнал отсутствует. При этом в этой же области при идентичных позициях лазерного луча наблюдался интенсивный сигнал на частоте оптических фононов кремния $520 \mathrm{~cm}^{-1}$, что свидетельствует о ненулевой толщине образца и исключает возможность ошибки позиционирования. Заметный сигнал $2000 \mathrm{~cm}^{-1}$ появляется только при удалении от края центрального отверстия более чем на 10 мкм, когда край лазерного луча начинает попадать на ДС. При дальнейшем перемещении лазерного луча в более толстую часть образца наблюдается быстрый рост интенсивности, который достигает максимума при удалении от края отверстия на 25 мкм. Пространственное положение максимума сигнала соответствует участку с минимальной толщиной фольги, при которой лазерный луч полностью попадает на ДС. При дальнейшем смещении возбуждающего луча от края образца наблюдается спад интенсивности КР сигнала $2000 \mathrm{~cm}^{-1}$, который обусловлен уменьшением интенсивности возбуждения в плоскости ДС вследствие поглощения луча, отраженного от обратной внутренней стороны фольги. Пространственные осцилляции сигнала КР хорошо согласуются с пространственным положением интерференционных полос на

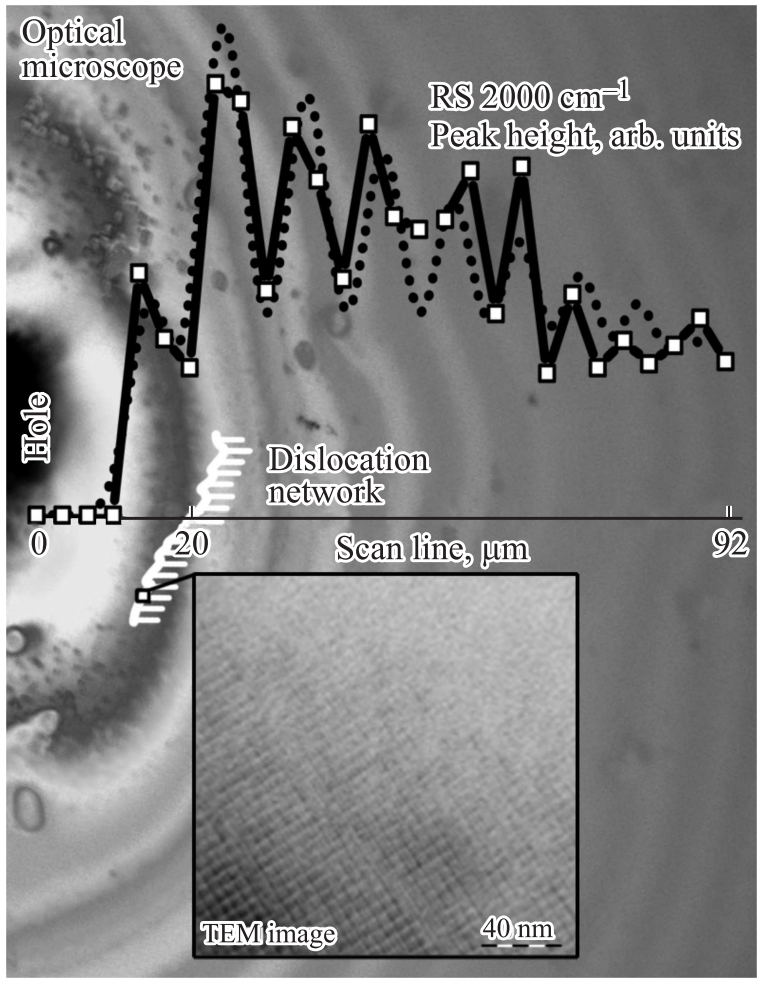

Рис. 2. Изображение исследуемой ПЭМ фольги в оптическом микроскопе с наложенным профилем распределения интенсивности линии КР $2000 \mathrm{~cm}^{-1}$ (сплошная линия). Пунктирная линия - теоретически рассчитанный профиль интенсивности пика $2000 \mathrm{~cm}^{-1}$. Белая гребенка показывает границу дислокационной сетки, определенной с помощью ПЭМ. На вставке пример ПЭМ изображения ДС в месте выхода ее на поверхность.

изображении, полученном в оптическом микроскопе, и обусловлены интерференцией на клиновидном образце.

Пунктирной линией на профиле интенсивности пика $2000 \mathrm{~cm}^{-1}$ (рис. 2) представлен результат расчета зависимости интенсивности возбуждающего света в плоскости ДС от места сканирования с учетом поглощения света, конечной ширины лазерной линии, а также интерференции, вызванной однократным отражением лазерного луча от внутренней поверхности кристалла. Для расчета использовались следующие параметры: коэффициент поглощения кремния на длине волны возбуждения КР $\alpha=0.7$ мкм $^{-1}$, показатель преломления $n=4$, глубина залегания ДС 150 нм и диаметр пятна лазерного луча 6 мкм. Как видно из рис. 2, обе кривые имеют черты, характерные только для сигнала от плоскости интерфейса, а именно отсутствие сигнала в области, где ДС нет, резкий рост интенсивности сигнала при приближении к ДС, достижение максимума на ДС и последующее убывание сигнала при увеличении толщины фольги. Отметим, что сигнал от любого источника рассеяния, равномерно распределенного по объему, должен характеризоваться монотонным ростом 


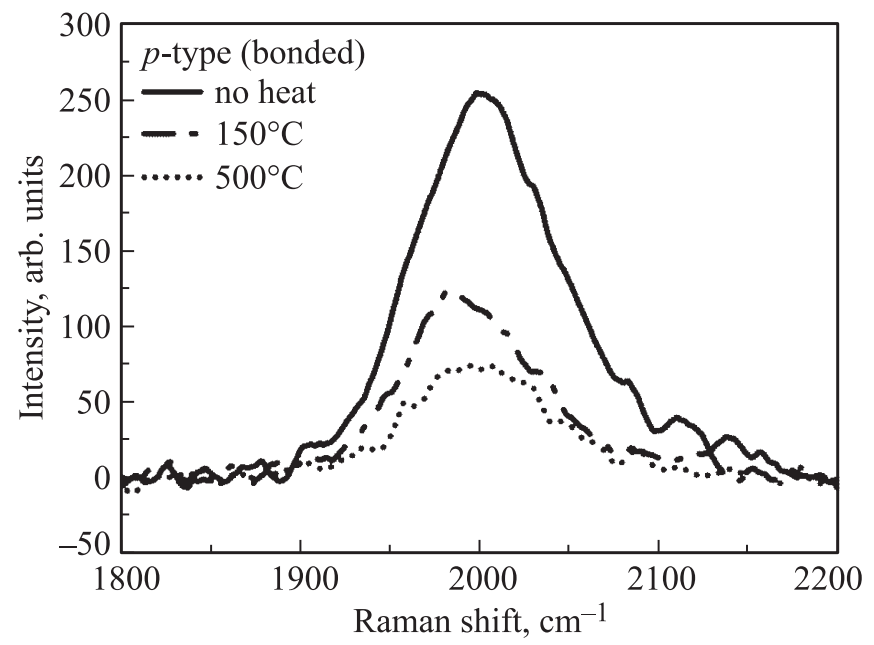

Рис. 3. Влияние часовых отжигов в вакууме на спектры КР в кремнии $p$-типа с ДС. Давление $10^{-5}$ Торр. (Приведенные спектры получены в одной точке, фон вычтен.)

интенсивности с интерференционной модуляцией, что, например, имело место в нашем случае для линии КР оптических фононов $520 \mathrm{~cm}^{-1}$ [20]. Таким образом, пик КР $2000 \mathrm{~cm}^{-1}$ однозначно связан с интерфейсом ДС, а его спектральное положение соответствует известной моде растяжения $\mathrm{Si}-\mathrm{H}$ связи $[21,22]$.

Другим дополнительным признаком водорода на дислокациях является его бо́льшая термическая стабильность, чем в регулярной решетке. Так, в упомянутых выше экспериментах по экзодиффузии $H$, в которых водород вводился в пластически деформированные образцы с помощью плазменной обработки при температурах около $300^{\circ} \mathrm{C}$, экстракция основной части водорода начиналась только при температурах выше $500^{\circ} \mathrm{C}$ и продолжалась вплоть до $800^{\circ} \mathrm{C}[11]$, в то время как в недеформированных образцах экзодиффузия полностью прекращалась уже при температурах ниже $450^{\circ} \mathrm{C}$.

На рис. 3 представлены результаты последовательных изохронных отжигов дислокационного образца $p$-типа при температурах 150 и $500^{\circ} \mathrm{C}$ в вакууме $\left(10^{-5}\right.$ Topp). Как видно из рисунка, отжиг при температуре $150^{\circ} \mathrm{C}$ привел к примерно двукратному уменьшению интенсивности пика $2000 \mathrm{~cm}^{-1}$. Кроме того, наблюдается небольшое смещение положения максимума в сторону меньших частот, что указывает на растяжение кремниевых связей [23], т.е. соответствует водороду, находящемуся в областях с большими растягивающими деформациями решетки - ближе к ядрам дислокаций.

После отжига при $500^{\circ} \mathrm{C}$ сигнал КР $2000 \mathrm{~cm}^{-1}$ еще несколько уменьшился, но все же значительная часть водорода (более четверти от первоначальной величины) осталась в окрестности интерфейса СП (рис. 3, пунктирная кривая). Этот факт находится в согласии с данными по высокотемпературной экзодиффузии из дислокационного кремния и свидетельствует о наличии сильно связанного с дислокациями водорода, для экс- тракции которого необходимы более высокие температуры и(или) более длительные времена отжигов. Таким образом, результаты исследований влияния отжига на спектры КР вблизи $2000 \mathrm{~cm}^{-1}$, а также его аномально большая ширина подтверждают предположение о наличии водорода, с различной энергией связи в окрестности ядра дислокации, следующее из теоретических расчетов $[13,14]$. В итоге мы идентифицировали уширенный пик $2000 \mathrm{~cm}^{-1}$ как проявление атомарного водородам на ВС в непосредственной окрестности от дислокаций.

До сих пор, говоря об моноатомном водороде в ВС положении, мы не заостряли внимание на его зарядовом состоянии, при том что, согласно теоретическим расчетам [24], для регулярной решетки энергии связи и колебательные частоты для $H^{+}$или $H^{0}$ крайне близки. Расчеты энергии связи водорода на ВС в полях напряжения дислокации [14], упомянутые выше, производились для электронейтрального атома водорода $\left(H^{0}\right)$, что, однако, было обусловлено прежде всего упрощением исходной модели. С другой стороны, экспериментальные работы [9], которые говорят об обнаружении положительного водорода $H^{+}$в ВС положении, прежде всего ссылаются на аналогию водорода с мюонием. Как результат, обнаруженный в спектре ИК поглощения для образцов, подверженных низкотемпературной протонной имплантации, пик $1998 \mathrm{~cm}^{-1}[8,9]$, был приписан к $H^{+}$. Учитывая хорошее совпадение положения данного пика с положением пика, который был обнаружен в настоящей работе, для нас представлял большой интерес получить дополнительные экспериментальные результаты, указывающие на зарядовое состояние водорода.

Малая глубина залегания ДС позволяет исследовать ее электрофизические свойства, так как она попадает в область пространственного заряда (ОПЗ) шотткидиода $[16,17]$. Если водород находится на ДС в заряженном состоянии, изменение заряда на интерфейсе СП вследствие его экстракции можно зарегистрировать с помощью измерений ВФХ.

Ранее неоднократно было показано, что при гидрогенизации из раствора плавиковой кислоты водород концентрируется в приповерхностной области кристалла, где пассивирует электрически активную примесь [1-4] и захватывается на ДС [25]. Водород не проникает глубже плоскости интерфейса СП, поскольку ДС препятствует диффузии водорода за ее пределы при гидрогенизации [16]. Экстракцию водорода с ДС можно вызвать в образцах $p$-типа с помощью отжига диода с приложенным обратным смещением (Reverse Bias Anneal/RBA), в ходе чего происходит диссоциация водородосодержащих комплексов и дрейф водорода на край ОПЗ [17]. Достигший края ОПЗ водород при понижении температуры до комнатных значений снова пассивирует акцепторы, что выражается в провале на профиле концентрации электрически активной примеси, определенном из ВФХ.

На рис. 4, а представлен пример профилей концентрации мелких электрически активных акцепторов до и после RBA для одного из исследуемых образцов. RBA 

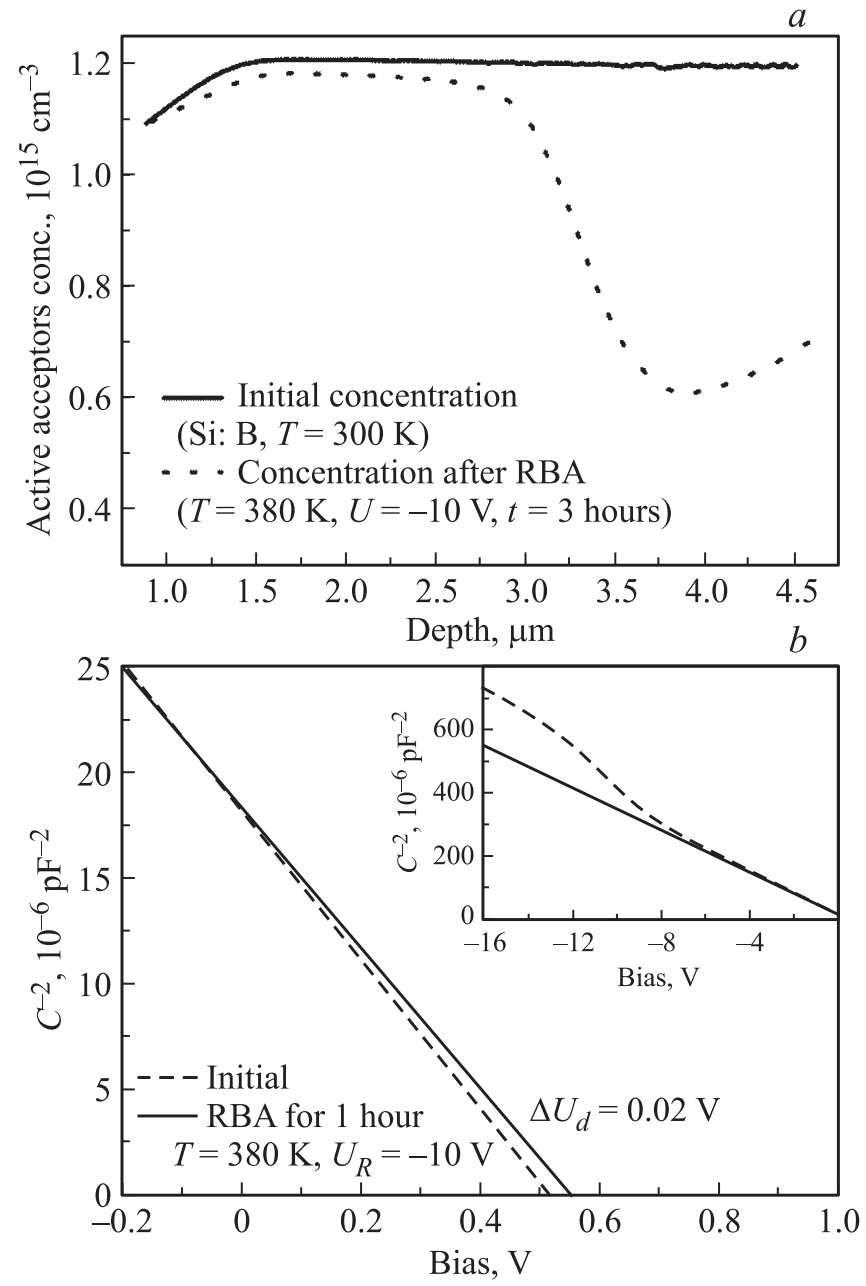

Рис. 4. $a-$ профиль концентрации некомпенсированной электрически активной примеси в дислокационном $p$-Si:B до и после RBA процедуры. $b-$ зависимость величины $C^{-2}$ от обратного смещения до и после RBA процедуры. Отсечки на оси смещения позволяют найти диффузионное напряжение.

процедура проводилась при температуре $380 \mathrm{~K}$ и обратном напряжении смещения $10 \mathrm{~B}$, длительность процедуры 3 ч. Интегрирование разницы профилей электрически активной примеси до и после RBA позволяет получить оценку величины экстрагированного из приповерхностной области водорода, которая оказалась равной $N_{\text {extr }}=1.2 \cdot 10^{11} \mathrm{~cm}^{-2}$. При этом в случае положительно заряженного водорода на ДС, его перемешение в глубь образца должно сопровождаться изменением величины диффузионного напряжения.

Из уравнения Пуассона следует соотношение для добавки диффузионного напряжения $\Delta V_{d}$, связанного с зарядом водорода на ДС и на акцепторах в слое образца между поверхностью и интерфейсом с ДС:

$$
\varepsilon \varepsilon_{0} \Delta V_{d}=e N_{\text {dis }} D+\frac{e N_{H-A}}{2} D^{2},
$$

где $N_{\text {dis }}$ - концентрация водорода на единицу площади ДС, $D$ - глубина залегания ДС от поверхности образ- ца, $N_{H-A}-$ концентрация положительно заряженного водорода на акцепторах в области между поверхностью образца и ДС. Если в приповерхностной области все акцепторы пассивированы водородом, то $N_{H-A}$ равна концентрации акцепторов $N_{A}=1.2 \cdot 10^{15} \mathrm{~cm}^{-3}$, и изменение диффузионного напряжения за счет экстракции с них водорода может быть оценено как 0.03 эВ. С другой стороны, экспериментально полученное количество экстрагированного водорода (рис. $4, a), N_{\mathrm{extr}}=1.2 \cdot 10^{11} \mathrm{~cm}^{-2}$, должно привести, с учетом заряда на ДС, к изменению $\Delta V_{d}=0.32$ B.

Изменение диффузионного напряжения можно определить по зависимости обратного квадрата емкости от напряжения, полученной из ВФХ. На рис. 4, $b$ представлен результат изменения диффузионного напряжения после проведения процедуры RBA. Получившееся значение $\Delta V_{d}=0.02 \mathrm{~B}$ оказалось в пределах погрешности совпадающим со значением, ожидаемым только для заряда протонов из объема приповерхностной области, но более чем на порядок меньше ожидаемого для водорода с ДС. Отсюда можно заключить, что заряд на ДС отсутствует, а значит, водород, находящийся в окрестности дислокаций, электронейтрален.

\section{4. Заключение}

Использование в настоящей работе в качестве образцов тонких фольг для ПЭМ позволило значительно повысить чувствительность регистрации КР и обнаружить пик КР при $2000 \mathrm{~cm}^{-1}$, который соответствует атомарному водороду и не проявляется в объемных образцах. Увеличения чувствительности удалось достичь за счет использования сильно поглощаемого возбуждающего света и благодаря интерференционному усилению накачки при снижении доли фонового сигнала за счет уменьшения толщины образца. Достигнутая чувствительность по количеству экстрагированного водорода для предложенного подхода в пересчете на единицу поверхности составляет величину порядка $\sim 10^{11} \mathrm{~cm}^{-2}$, что соответствует абсолютному числу атомов, попадающих в пятно луча лазера в несколько десятков тысяч.

Незначительное изменение диффузионного напряжения после RBA процедуры свидетельствует о нейтральности водорода у дислокаций и позволяет приписать пик КР в окрестности $2000 \mathrm{~cm}^{-1}$ электронейтральному водороду $H^{0}$ в ВС конфигурации.

Устойчивость обнаруженной линии КР к отжигу при столь высокой температуре как $500^{\circ} \mathrm{C}$ подтверждает существование водорода в окрестности дислокаций, который сильнее связан с кремнием, чем в регулярной решетке. Данный факт находится в качественном согласии с данными теоретических расчетов [14], которые предсказывают как устойчивость и сильную связь водорода в ядрах дислокаций, так и предпочтительность атомарной формы водорода в полях упругих напряжений вокруг дислокаций. Также, согласно [14], энергия водорода в 
решетке при изменении расстояния до ядра дислокации, $r$, может быть аппроксимирована как $-2.2 / r$ (эВ/А), что должно приводить к значительной дисперсии энергий его связи, что в свою очередь объясняет аномально большую ширину обнаруженного пика.

Работа выполнена при частичной поддержке гранта Министерства образования и науки 14.В25.31.0017. Исследования проведены с использованием оборудования ресурсных центров МРЦ по направлению „Нанотехнологии“ и „Оптические и лазерные методы исследования вещества“" Научного парка СПбГУ.

Авторы выражают свою благодарность О. Конончуку на предоставленные образцы и Е. Борисову за проведение измерений спектров КР.

\section{Список литературы}

[1] J. Pankove, D. Carlson, J. Berkeyheiser, R. Wance. Phys. Rev. Lett., 51, 2224 (1983).

[2] C.-T. Sah. Appl. Phys. Lett., 43, 204 (1983).

[3] N.M. Johnson, C. Herring, D.J. Chadi. Phys. Rev. Lett., 56, 769 (1986).

[4] S.J. Pearton, J.W. Corbett, T.S. Shi. Appl. Phys. A Mater., 43, 153 (1987).

[5] K. Chang, D. Chadi. Phys. Rev. B, 40, 11644 (1989).

[6] G.G. Van de Walle. Phys. Rev. Lett., 80, 2177 (1998).

[7] A.W.R. Leitch, V. Alex, J. Weber. Phys. Rev. Lett., 81, 421 (1998).

[8] H.J. Stein. J. Electron. Mater., 4, 159 (1975).

[9] M. Budde, G. Lupke, C.P. Cheney, N.H. Tolk, L.C. Feldman. Phys. Rev. Lett., 85, 1452 (2000).

[10] M. Budde, C.P. Cheney, G. Lupke, N.H. Tolk, L.C. Feldman. Phys. Rev. B, 63, 195203 (2001).

[11] V.V. Kveder, R. Labusch, Y.A. Ossipyan. Phys. Status Solidi A, 84, 149 (1984).

[12] C. Kisielowski-Kemmerich, W. Beyer. J. Appl. Phys., 66, 552 (1989).

[13] M. Matsubara, J. Godet, L. Pizzagalli. Phys. Rev. B, 82, 024107 (2010).

[14] M. Matsubara, J. Godet, L. Pizzagalli. J. Phys. Condens. Matter, 22, 035803 (2010).

[15] M.I. Heggie, S. Jenkins, C.P. Ewels, P. Jemmer, R. Jones, P.R. Briddon. J. Phys. Condens. Matter, 12, (49), 10263 (2000).

[16] A. Loshachenko, A. Bondarenko, O. Vyvenko, O. Kononchuk. Phys. Status Solidi C, 10, 36 (2013).

[17] A. Loshachenko, O. Vyvenko, O. Kononchuk. Sol. St. Phenomena, 205-206, 341 (2014).

[18] I. Isakov, A. Bondarenko, O. Vyvenko, V. Vdovin, E. Ubyivovk, O. Kononchuk. J. Phys. Conf. 281, 012010 (2011).

[19] V.I. Vdovin, E.V. Ubyivovk, O.F. Vyvenko. Semiconductors, 47, 264 (2013).

[20] N. Vysotskii, A. Loshachenko, E. Borisov, O. Vyvenko. J. Phys. Conf. 690, 012004 (2016).

[21] C.G. Van de Walle, P.J.H. Denteneer, Y. Bar-Yam, S.T. Pantelides. Phys. Rev. B, 39, 10791 (1989).

[22] K.J. Chang, D.J. Chadi. Phys. Rev. B, 40, 11644 (1989).
[23] C.-Y. Peng, C.-F. Huang, Y.-C. Fu, Y.-H. Yang, C.-Y. Lai, S.-T. Chang, C.W. Liu. J. Appl. Phys. 105, 083537 (2009).

[24] S.K. Estreicher. Mater. Sci. Eng. R., 14, 319 (1995).

[25] Y. Park, J. Lu, G. Rozgonyi. Electron. Mater. Lett., 6, 1 (2010).

Редактор Г.А. Оганесян

\section{Atomic configuration and charge state of hydrogen at dislocations in silicon}

\section{N.V. Vysotskii, A.S. Loshachenko, O.F. Vyvenko}

\section{St. Petersburg State University, 198504 St. Petersburg, Russia}

Abstract The impact of hydrogenation on vibrational spectra and electrophysical properties of the samples with dislocations network (DN) produced by silicon wafer bonding has been studied. To increase measurement sensitivity and to separate DNrelated signal in Raman spectra (RS) thin foils conventionally prepared for transmission electron microscopy (TEM) were used. In DN samples Raman peak at $2000 \mathrm{~cm}^{-1}$ was found which was absent in dislocation-free samples and survives after $500^{\circ} \mathrm{C}$ annealing. Comparison of the obtained experimental data with the results of recent theoretical calculations allowed to ascribed the RS peak to $H^{0}$ in $\mathrm{Si}-\mathrm{Si}$ bond center which is metastable in the ideal $\mathrm{Si}$ lattice but stabilizes at dislocations. 